\title{
Augmenting Experimental Data with Simulations to Improve Activity Classification in Healthcare Monitoring
}

\author{
Chong Tang*, Shelly Vishwakarma*, Wenda $\mathrm{Li}^{*}$, Raviraj Adve ${ }^{\dagger}$, Simon Julier ${ }^{\ddagger}$, Kevin Chetty* \\ ${ }^{*}$ Department of Security and Crime Science, University College London, UK \\ ${ }^{\ddagger}$ Department of Computer Science, University College London, UK \\ ${ }^{\dagger}$ Department of Electrical and Computer Engineering, University of Toronto, Canada \\ \{chong.tang.18, s.vishwakarma, wenda.li, s.julier, k.chetty\}@ucl.ac.uk, rsadve@comm.utoronto.ca
}

\begin{abstract}
Human micro-Doppler signatures in most passive WiFi radar (PWR) scenarios are captured through real-world measurements using various hardware platforms. However, gathering large volumes of high quality and diverse real radar datasets has always been an expensive and laborious task. This work presents an open-source motion capture data-driven simulation tool SimHumalator that is able to generate human microDoppler radar data in PWR scenarios. We qualitatively compare the micro-Doppler signatures generated through SimHumalator with the measured real signatures. Here, we present the use of SimHumalator to simulate a set of human actions. We demonstrate that augmenting a measurement database with simulated data, using SimHumalator, results in an $8 \%$ improvement in classification accuracy. Our results suggest that simulation data can be used to augment experimental datasets of limited volume to address the cold-start problem typically encountered in radar research.
\end{abstract}

Index Terms-Passive WiFi Sensing, micro-Dopplers, activity recognition, deep learning, simulator

\section{INTRODUCTION}

Indoor sensing applications such as assisted living (fall detection in older people), bio-medical surveillance (monitoring patients), and smart home such as hand gesture recognition have used intrusive systems such as bulky wearable sensors and expensive camera systems. Some of these sensors, however, are restricted in the sense that they can operate only under suitable environmental conditions. For instance, cameras can work only in the line of sight conditions and thus have limited coverage [1], [2]. On the other hand, the applications of wearable sensors are largely affected by perceived privacy risk, comfort level, battery life and so on [3].

WiFi based passive radar systems have attracted considerable attention for indoor monitoring. The approach can leverage existing WiFi (or other wireless) communications to passively capture radar returns. Therefore, with high monitoring sensitivity, these systems are less complicated and have lower energy consumption. Furthermore, passive WiFi radar (PWR) systems can gather time-varying radar returns due to the variable speeds of human body parts'. The information's remarkable advantages are that it contains the overall motion tendency and additional frequency modulation induced by the body's micro-movements (popularly known as micro-Dopplers).

A typical PWR system consists of two antennas - one acts as a reference to capture direct WiFi transmissions. The second is a surveillance antenna to capture signals reflected off targets in the propagation environment. The direct signal is cross-correlated with the echoes reflected from the target to extract micro-Doppler information. Micro-Doppler signatures have been proved to have excellent prospects for indoor healthcare applications such as fall detection [4], [5], respiratory monitoring [6], and the debilitating motor symptoms of Parkinson's patients [7].

More recently, there is an active thrust towards using deep learning assisted solutions for activity recognition using micro-Doppler data in passive radar-based sensing scenarios [8]. Training deep learning algorithms requires a vast amount of good quality data. However, collecting real-world micro-Doppler data can be a very laborious and costly task. Additionally, the signatures are significantly affected by different environmental conditions, sensor parameters, and target characteristics. Therefore, the volumes of data captured are often limited and unbalanced, significantly affecting deep learning algorithms' performance.

In this work, we present an open-source simulation tool for PWR, called SimHumalator [9]. We leverage human micro-Doppler data generated using SimHumalator to augment measured data. The outcomes highlight that for cases in which only limited experimental data is available for training, classification performance can be improved. In experiments, data from both marker-based and marker-less systems are available for SimHumalator. In [9], we reported on capturing human animation data using a marker-based system called Phase-Space. Here, we use a cheaper, markerless, sensor called Kinect v2 to track the human body skeleton. We captured animation data from three human subjects undergoing six different activities directly related to healthcare applications. We used this animation and combined it with the IEEE 802.11g standard WiFi transmissions generated through the Matlab WLAN toolbox [10] to simulate the reflected signals.

SimHumalator can generate micro-Doppler radar returns 
as a function of target characteristics (the aspect of the target to the radar), sensor parameters (waveform, radar configurations such as monostatic, bistatic-in line, and bistatic circular), and radar signal processing parameters (coherent processing interval (CPI), pulse repetition interval (PRI)). However, here, we have restricted our measurements to simple monostatic scenarios with fixed target and sensor parameters. We qualitatively compare the radar signatures generated through two synchronized systems- a real PWR system and motion capture Kinect v2 sensor.

Our results demonstrate that there is a close similarity between both the measured spectrograms and the simulated spectrograms. Measured spectrograms, as expected, are noisy compared to the simulated ones. Real signatures encounter environmental factors such as noise, multipath, and propagation loss due to the dynamic propagation medium. We have done a detailed augmentation study in different classification scenarios. To sum up, our contributions in this paper are the following:

1) We leverage simulation data provided by SimHumulator for data augmentation to solve the practical problem of insufficient or unbalanced micro-Doppler training data.

2) We propose two data augmentation methods- Replacement and Augmentation and validate their performance in different scenarios using the deep neural network VGG16.

We observe an increase in average classification accuracies up to $8 \%$. It shows that the simulation data can ease the limitation associated with the smaller real databases.

Our paper is organized as follows. Section II describes the setup of the experimental environment, and the frameworks of SimHumalator and PWR system are introduced, respectively. It also describes the data collection in different PWR scenarios and presents the simulation data's performance by comparison. Section III then presents interesting classification scenarios where we augment measurement data with simulation data and report the resulting classification performances. We finally conclude our paper in Section IV.

\section{EXPERImental Setup AND SYSTEM FrameWORK}

In this section, we present our experimental setup comprising two synchronized systems: a PWR system and a Kinect v2 sensor, as shown in Fig 1 . We set up the PWR system in a monostatic configuration. We restricted our measurements to direct line-of-sight conditions between $0.8 \mathrm{~m}$ to $3.8 \mathrm{~m}$. A Kinect v2 sensor uses infrared depth cameras to track the three-dimensional location of the 25 joints on the human body. Kinect v2 is a marker-less motion capture technology [11], [12]. The measured data

\footnotetext{
${ }^{1}$ We believe that the motion capture data-driven SimHumalator can effectively generate high-quality micro-Doppler spectrograms and support the smaller real databases. Adding environmental factors into the simulations will be part of future research. SimHumalator can be freely downloaded from https://uwsl.co.uk/.
}

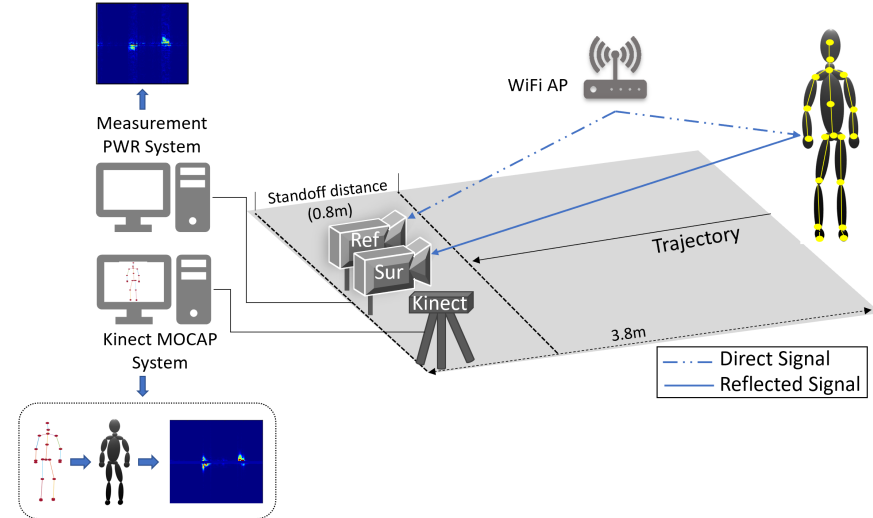

Fig. 1: The experimental setup comprising two synchronized systems- a PWR system and a Kinect v2 sensor- collects radar data and motion capture data simultaneously.

is then processed to generate spectrograms showing the corresponding micro-Doppler signature.. We will describe the signal processing methodology briefly in the subsequent sections.

\section{A. Simulation Framework}

We adopt a simulation framework similar to the one presented in [9]. Based on the Kinect v2 sensor, we simulate a typical IEEE $802.11 \mathrm{~g}$ WiFi signal using MATLAB's WLAN toolbox in the $2.4 \mathrm{GHz}$ band with a channel bandwidth of $B W=20 \mathrm{MHz}$ and human animation data. The simulated baseband signal $y_{T}(t)$ shown in (1), comprises of a continuous stream of $P$ transmission packets each of duration $T_{P R I}$ sec. $T_{P R I}$ corresponds to the pulse repetition interval.

$$
x_{T}(t)=\frac{1}{\sqrt{(} P)} \sum_{p=1}^{P} \sum_{n=1}^{N} y_{T}[n] h_{T}\left(t-n t_{s}-p T_{p}\right)
$$

Here, $N$ is the total number of time-domain samples, $t_{s}=$ $(1 / B W)=50 n$ sec is the sample period and $h_{T}(t)$ is the pulse shaping filter. We finally up-convert the baseband signal for transmission at a carrier frequency of $f_{c}=2.4 \mathrm{GHz}$ to obtain

$$
X_{T}(t)=x_{T}(t) e^{-j 2 \pi f_{c} t}
$$

In order to generate the reflected signal, we time-delayed the generated WiFi signal by $t_{b}$ and Doppler-shifted it by $f_{D b}$ according to human motion characteristics resulting in the received signal

$$
X_{R}(t)=\sum_{p=1}^{P} \sum_{b=1}^{B} \sigma_{b}(t) X_{T}\left(t-t_{b}-p T_{p}\right) e^{-j 2 \pi f_{D b} p T_{p}}+n(t)
$$

Human motion can be modelled as a nonrigid body consisting of a combination of radar scattering centers. We use animation data captured by Kinect v2 to gather the time-varying three-dimensional positions of 25 joints on the human body. We assume the scattering centers to be at the middle of the bones and, compute the reflectivity $\sigma_{b}(t)$, of each, scatter taking into account the aspect angle and the bone's location with respect to the radar. 
We cross-correlate the direct WiFi signal, and the surveillance signal to generate the cross ambiguity functions (CAFs). Since the bandwidth of a WiFi signal is limited to $20 \mathrm{MHz}$, the range resolution to locate targets in indoor scenarios is not sufficient. In contrast, the Doppler resolution, which is defined by the integration time $T_{i}$ as $\Delta f_{d}=1 / T_{i}$, can be adjusted to allow the desired Doppler resolution required to detect human activity. The CAF process can be written as:

$$
C A F\left(\tau, f_{d}\right)=\int_{0}^{T_{i}} X_{R}(t) X_{T}^{*}(t-\tau) e^{j 2 \pi f_{D b} t} d t
$$

Where $*$ denotes complex conjugation.

Since we focus on the Doppler information, we can suppress the direct signal interference using the CLEAN algorithm [13]. The CLEAN algorithm calculates the selfCAF based only on the reference signal and subtracts it from the CAF calculated in (4). We refer the readers to [9] for a detailed description of the signal processing and simulation framework.

\section{B. PWR System Framework}

In PWR system setup, two $14 \mathrm{dbm}$ Yagi antennas had been used. One was used for reference channel, and another one was used for surveillance channel. Two NI USRP-2921 [14] were used for real-time signal acquisition. The WiFi measurement and animation data were measured together from three participants including six different activities: sitdown, stand-up, sit-to-walk, walk-to-sit, walk to fall-down, and stand-from-floor to walk. Each activity was completed within 5-10 seconds and repeated 20 times, resulting in 60 measurements for each activity.

Overall, we gathered 360 measurement data and generated 360 simulated data over six activities.

\section{Spectrograms Evaluation}

In this section, we present a qualitative comparison between the micro-Doppler spectrograms generated using SimHumalator, the PWR measurement system, and the animation data velocity-time profiles. Fig.2 (a)-(c) shows the velocity-time profile (generated using motion capture data), and simulated and the measured spectrograms respectively for a human undergoing a motion of first sitting down on a chair followed by standing up from the chair. The qualitative similarity between all the signatures is evident from the figure. As the human sits down, we observe negative Doppler due to the bulk body motion. The positive microDoppler arises due to arm motion and legs moving slightly in the radar direction while sitting down. However, the strength of these micro-Dopplers is low compared to bulk body motion. After a 5sec delay, the human subject stands up from the chair, resulting in mostly positive Dopplers. Similarly, Fig.2 (d)-(f) presents spectrograms corresponding to a human standing up from a chair and prior to walking. Fig.2 (g)-(i), presents spectrograms corresponding to a human transitioning from walking to falling. The strength of the signals of the spectrograms shown in Fig.2 (a)(i) may not be the same; however, the envelope of the velocity-time profile is visually very similar across all. Our simulations do not synthesize environmental factors like noise, propagation loss, and multipath. Therefore, the simulated spectrograms are clean compared to the measured signatures, which are noisier and have lower reflectivity.

\section{EXPERIMENTAL RESUlTS AND ANALYSIS}

In this section, we present classification results from various experimental scenarios. We first tested the classification algorithm's performance when measurement data was used for both training and test scenarios (TMTM). Next, the training dataset was created by combining both the simulation and experimentally measured data. During the training process, we used only unseen measurement data to test the pre-trained VGG16 [15] model's performance. We reshaped the size of input spectrograms into 224 by 224; the batch size is set to 64 . The learning rate and momentum of the stochastic gradient descent optimizer are set to 0.01 and 0.9 , respectively; the output shape is modified to 6; for our multi-class classification task, the loss function is categorical cross-entropy; the epoch is set to 150. Moreover, we repeated each training 15 times and then summarized the model with the mean of resulting accuracy. The data was used to assess the Replacement and Augmentation schemes for generating training data

\section{A. Replacement Study}

Replacement refers to replacing part of the measurement data in the original training set with the simulation data. The scheme can achieve data augmentation by replacing unbalanced or low-quality measurement data. As shown in Fig. 3, we split the total measurements data $(M)$ into training set of size $m M$ and testing set of size $(1-m) M$, and then fixed the size of the training set. Next, we used the replacement ratio within the training set as $s$ to replace parts of the measurement data with the same classes' simulation data. Therefore, simulation data of size $s m M$ in the training set, i.e., measurement data of size $M-s m M$, is retained.

In the replacement experiments, we varied both replacement percentage of $s$ as well as the real measurement data split ratio $m$. We varied $m$ from $30 \%$ to $70 \%, s$ from $10 \%$ to $90 \%$ and report the resulting classification accuracies for VGG16 in TABLE I. We can draw the following inferences from the results:

1) Outcome 1: For the same $s$, that is when the percentage of simulation data replacement is fixed, the classification accuracies increase with the increase in $m$ (true measurement dataset size). This shows the negative impact of insufficient training on the classification results. Moreover, the results highlights the utility of augmentation techniques for addressing issues around limited training data in e-healthcare and ambient assisted living radar applications 

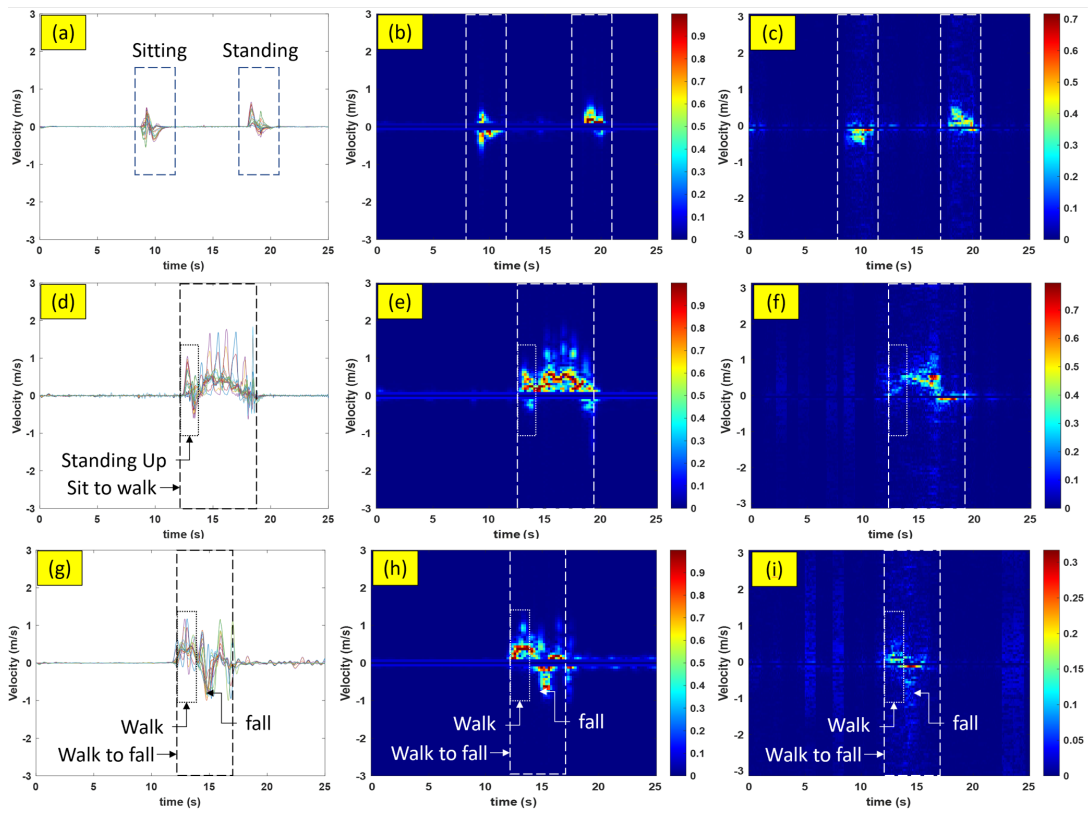

Fig. 2: Velocity-time profile using motion capture data, simulated and measured Doppler spectrograms of a human undergoing (a)-(c) sitting followed by standing up motion, (d)-(f) stand up from chair and starts walking, (g)-(i) walk to fall motion respectively.

2) Outcome 2: For the same $m$, the classification accuracies decrease with an increase of $s$. This indicates that when the measurement data is replaced with more simulation data, the performances drop. This can be attributed to the fact that there exist differences between measurement data and simulated data due to various environmental factors. So, only a certain amount of corrupted measurement data can be replaced with simulation data. We do note that this could be because the measurement scenario involved repeated measurements of the same activity in the same environment.

3) Outcome 3: Under the same $m$, specific $s$ can improve the classification accuracy. Especially when we have a smaller size of the training set, the improvement effect is better. The most obvious one is that when $m=30 \%$ and $s=10 \%$, we can observe that the replacement can improve the classification accuracy by about $8 \%$ compared to TMTM's accuracy. This is the case when we already have limited measurement training data support.

We can finally conclude that when there is a sufficient amount of the measurement data for training (typically at least more than 50\%), the replacement scheme for data augmentation can effectively improve classification accuracy. However, environmental factors impact the measurement data and have not yet been incorporated into out simulation platform. Therefore, if the high $s$ is used for training DNN and then to test the measurement data, these environmental factors are not effectively considered by the DNN, resulting in a decrease in classification accuracy. Therefore in the replacement augmentation scheme, s must be chosen carefully.

\section{B. Augmentation Study}

Unlike the case of replacement, the augmentation scheme adds additional simulation data increase the overall size of the training dataset. The study can be summarized in Fig. 4. The total measurement data $(M)$ is divided into the training set and the test set according to the split ratio of $m$. Then we added the $s$ percentage of the total simulation data $(S)$ into the training set, resulting in a training set size of $(m M+s S)$.

We used the VGG16 network and report the classification results for the augmentation study in TABLE II. The following results are obtained from the analysis:

1) Under the same $s$, the classification accuracies increase with an increase of $m$.

2) Under the same $m$, even if we varied the $s$ from $0 \%$ (i.e., TMTM) to $100 \%$, the classification accuracies are relatively close to each other, and importantly almost all results are better than the case when only measurement data was used for training the neural network.

3) The smaller $m$, the more pronounced the improvement.

Among them, phenomena 1 and 3 show similar characteristics, as we observed in the replacement scheme. However, for phenomenon 2, the classification accuracies no longer deteriorate with an increase of $s$. The possible reason is that the simulation samples add up to the measurement samples instead of replacing them. This results in a training set having sufficient environmental features and also motion patterns to train a DNN. 


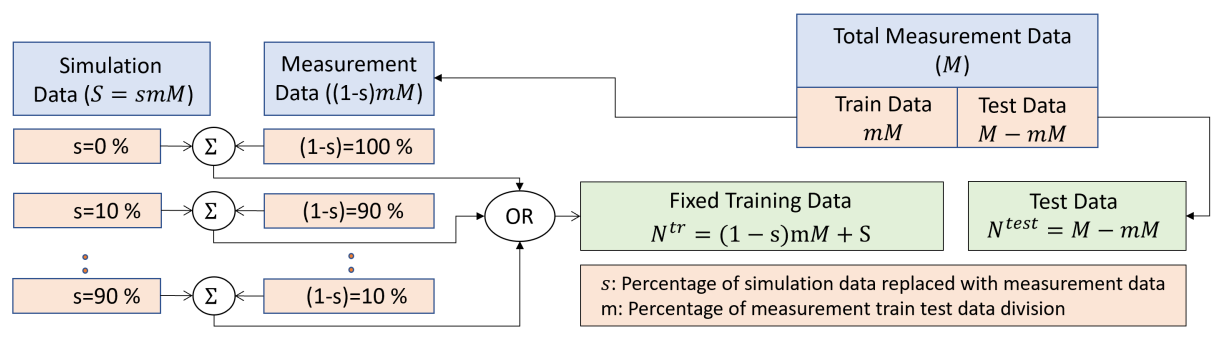

Fig. 3: Replacement study: The training dataset remains fixed. The ratio of simulation data to measurement data is varied to study the impact of data replacement on the classification performance. $s=0$, is a special case where both training and test data comprise of measured spectrograms only.

TABLE I: Classification accuracy results for replacement study. $\mathrm{m}$ is varied from $30 \%$ to $70 \%$. $\mathrm{s}$ is varied from $0 \%$ to $90 \%$. $\mathrm{s}=0$, is a special case where both training and test data comprise of measured spectrograms only (TMTM).

\begin{tabular}{|c|c|c|c|c|c|c|}
\hline \multicolumn{2}{|c}{$\begin{array}{c}\text { Measurement } \\
\text { Train/Test ratio (m) }\end{array}$} & $30 \%-70 \%$ & $40 \%-60 \%$ & $50 \%-50 \%$ & $60 \%-40 \%$ & $70 \%-30 \%$ \\
\hline \multicolumn{2}{|c|}{ TMTM } & $86.1 \%$ & $95.2 \%$ & $96.8 \%$ & $97.5 \%$ & $98.1 \%$ \\
\hline Replacement & $90 \% \mathrm{~m}-10 \% \mathrm{~s}$ & $94.2 \%$ & $97.5 \%$ & $97.4 \%$ & $97.6 \%$ & $98.6 \%$ \\
\hline & $80 \% \mathrm{~m}-20 \% \mathrm{~s}$ & $92.4 \%$ & $97.4 \%$ & $97.1 \%$ & $97.5 \%$ & $97.9 \%$ \\
\hline & $70 \% \mathrm{~m} / 30 \% \mathrm{~s}$ & $90.6 \%$ & $95.7 \%$ & $96.5 \%$ & $96.4 \%$ & $97.4 \%$ \\
\hline & $60 \% \mathrm{~m} / 40 \% \mathrm{~s}$ & $89.8 \%$ & $94.6 \%$ & $94.3 \%$ & $95.1 \%$ & $97.2 \%$ \\
\hline & $50 \% \mathrm{~m} / 50 \% \mathrm{~s}$ & $83.3 \%$ & $90.4 \%$ & $90.1 \%$ & $91.1 \%$ & $93.1 \%$ \\
\hline & $40 \% \mathrm{~m} / 60 \% \mathrm{~s}$ & $77.2 \%$ & $85.7 \%$ & $85.9 \%$ & $88.9 \%$ & $92.8 \%$ \\
\hline & $30 \% \mathrm{~m} / 70 \% \mathrm{~s}$ & $75.3 \%$ & $79.7 \%$ & $83.2 \%$ & $86.3 \%$ & $88.8 \%$ \\
\hline & $20 \% \mathrm{~m} / 80 \% \mathrm{~s}$ & $72.9 \%$ & $69.8 \%$ & $77.7 \%$ & $76.8 \%$ & $81.4 \%$ \\
\hline & $10 \% \mathrm{~m} / 90 \% \mathrm{~s}$ & $63.7 \%$ & $62.2 \%$ & $68.6 \%$ & $66.0 \%$ & $69.8 \%$ \\
\hline
\end{tabular}

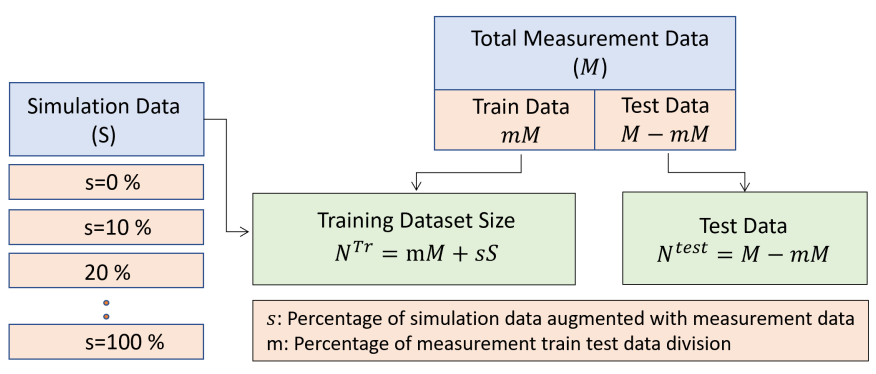

Fig. 4: Augmentation study: The training dataset changes with the percentage of simulated data (s) augmented with the measurement training data. The percentage of simulation data is varied to study the impact of data augmentation on the classification performance. $\mathrm{s}=0$, is a special case where both training and test data comprise of measured spectrograms only.

We have also compared the TMTM's performance with the corresponding $s=100 \%$ results in Fig. 5 . We can observe that after augmentation, all classification accuracies can reach more than $94 \%$ and are better than the original TMTM's accuracies. In particular, when $m=30 \%$, the accuracy can be improved by over $8 \%$.

\section{CONCLUSION}

This paper presents an effective method to augment measured micro-Doppler spectrograms with the simulated radar data. We introduced SimHumalator and our PWR measurement system. Next, we make a qualitative comparison between the simulated spectrogram, the measured spectrogram, and the ground truth velocity information. From this, we can conclude that SimHumalator can effectively

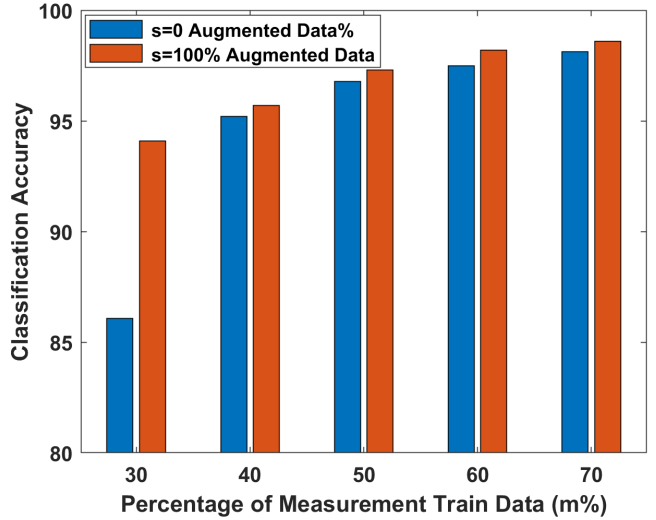

Fig. 5: Augmentation study: Classification accuracies of percentage of measurement data augmented with simulation data $(s=100 \%)$.

generate high-quality micro-Doppler spectrograms from the motion capture data, which is essential for the data augmentation. We proposed two augmentation schemes under different training and test conditions and evaluated the classification performance of a DNN is each scenario. In both schemes, we can achieve a performance improvement up to $8 \%$. The smaller the volume of measured data in the training set, the more pronounced the improvement in classification accuracy. This has vital practical significance. However, we can also find differences. For the replacement study, the classification accuracy deteriorates with the increase of the replacement ratio. Therefore, this scheme might be more suitable for replacing a small amount of low-quality measured data and filling a small number of 
TABLE II: Classification accuracy results for augmentation study. $\mathrm{m}$ is varied from $30 \%$ to $70 \%$. $\mathrm{s}$ is varied from $0 \%$ to $100 \%$. $\mathrm{s}=0$, is a special case where both training and test data comprise of measured spectrograms only (TMTM).

\begin{tabular}{|c|c|c|c|c|c|c|}
\hline \multicolumn{2}{|c|}{$\begin{array}{c}\text { Measurement } \\
\text { Train/Test ratio (m) }\end{array}$} & $30 \%-70 \%$ & $40 \%-60 \%$ & $50 \%-50 \%$ & $60 \%-40 \%$ & $70 \%-30 \%$ \\
\hline \multicolumn{2}{|c|}{ TMTM } & $86.1 \%$ & $95.2 \%$ & $96.8 \%$ & $97.5 \%$ & $98.1 \%$ \\
\hline Augmentation & $100 \% \mathrm{~m}+10 \% \mathrm{~s}$ & $93.0 \%$ & $97.4 \%$ & $97.6 \%$ & $99.1 \%$ & $98.7 \%$ \\
\hline & $100 \% m+20 \% s$ & $94.2 \%$ & $97.5 \%$ & $97.7 \%$ & $98.2 \%$ & $98.5 \%$ \\
\hline & $100 \% \mathrm{~m}+30 \% \mathrm{~s}$ & $93.7 \%$ & $97.7 \%$ & $97.5 \%$ & $99.1 \%$ & $98.5 \%$ \\
\hline & $100 \% \mathrm{~m}+40 \% \mathrm{~s}$ & $93.7 \%$ & $97.9 \%$ & $97.7 \%$ & $99.1 \%$ & $98.4 \%$ \\
\hline & $100 \% \mathrm{~m}+50 \% \mathrm{~s}$ & $94.4 \%$ & $97.9 \%$ & $97.4 \%$ & $99.1 \%$ & $98.3 \%$ \\
\hline & $100 \% \mathrm{~m}+60 \% \mathrm{~s}$ & $94.3 \%$ & $97.2 \%$ & $97.6 \%$ & $95.5 \%$ & $98.5 \%$ \\
\hline & $100 \% \mathrm{~m}+70 \% \mathrm{~s}$ & $93.6 \%$ & $97.7 \%$ & $98.0 \%$ & $97.3 \%$ & $98.5 \%$ \\
\hline & $100 \% \mathrm{~m}+80 \% \mathrm{~s}$ & $94.0 \%$ & $96.7 \%$ & $97.6 \%$ & $96.4 \%$ & $98.3 \%$ \\
\hline & $100 \% \mathrm{~m}+90 \% \mathrm{~s}$ & $93.8 \%$ & $96.5 \%$ & $97.3 \%$ & $99.1 \%$ & $98.5 \%$ \\
\hline & $100 \% \mathrm{~m}+100 \% \mathrm{~s}$ & $94.1 \%$ & $95.7 \%$ & $97.3 \%$ & $98.2 \%$ & $98.6 \%$ \\
\hline
\end{tabular}

imbalanced classes in practical applications. On the contrary, when the training set can provide relatively sufficient measurement features, the augmentation scheme's classification results can achieve better accuracy under any mixing conditions. So generally, we can prioritise the use of this scheme.

Overall, the paper demonstrates the feasibility of using simulated micro-Doppler spectrograms generated by SimHumalator for data augmentation tasks. Future research will incorporate environmental factors into the SimHumalator. We expect that even with limited environmental features, augmenting the training data by SimHumalator will improve the DNN performance.

\section{ACKNOWLEDGMENTS}

This work is part of the OPERA project funded by the UK Engineering and Physical Sciences Research Council (EPSRC), Grant No: EP/R018677/1.

\section{REFERENCES}

[1] C.-H. Chen, J. Favre, G. Kurillo, T. P. Andriacchi, R. Bajcsy, and R. Chellappa, "Camera networks for healthcare, teleimmersion, and surveillance," Computer, vol. 47, no. 5, pp. 26-36, 2014.

[2] G. Diraco, A. Leone, and P. Siciliano, "An active vision system for fall detection and posture recognition in elderly healthcare," in 2010 Design, Automation \& Test in Europe Conference \& Exhibition (DATE 2010). IEEE, 2010, pp. 1536-1541.

[3] X. Wang, L. White, X. Chen, Y. Gao, H. Li, and Y. Luo, "An empirical study of wearable technology acceptance in healthcare," Industrial Management \& Data Systems, 2015.

[4] B. Erol and M. G. Amin, "Radar data cube analysis for fall detection," in 2018 IEEE International Conference on Acoustics, Speech and Signal Processing (ICASSP). IEEE, 2018, pp. 2446-2450.

[5] B. Erol, S. Z. Gurbuz, and M. G. Amin, "Motion classification using kinematically sifted acgan-synthesized radar micro-doppler signatures," IEEE Transactions on Aerospace and Electronic Systems, 2020.

[6] Q. Chen, Y. Liu, B. Tan, K. Woodbridge, and K. Chetty, "Respiration and activity detection based on passive radio sensing in home environments," IEEE Access, vol. 8, pp. 12 426-12 437, 2020.

[7] X. Yang, S. A. Shah, A. Ren, N. Zhao, Z. Zhang, D. Fan, J. Zhao, W. Wang, and M. Ur-Rehman, "Freezing of gait detection considering leaky wave cable," IEEE Transactions on Antennas and Propagation, vol. 67, no. 1, pp. 554-561, 2018.

[8] W. Li, B. Tan, and R. Piechocki, "Passive radar for opportunistic monitoring in e-health applications," IEEE journal of translational engineering in health and medicine, vol. 6, pp. 1-10, 2018.

[9] S. Vishwakarma, W. Li, C. Tang, K. Woodbridge, R. Adve, and K. Chetty, "Simhumalator: An open source wifi based passive radar human simulator for activity recognition."
[10] MATLAB, 9.7.0.1190202 (R2019b). Natick, Massachusetts: The MathWorks Inc., 2018.

[11] A. D. Singh, S. S. Ram, and S. Vishwakarma, "Simulation of the radar cross-section of dynamic human motions using virtual reality data and ray tracing," in 2018 IEEE Radar Conference (RadarConf18). IEEE, 2018, pp. 1555-1560.

[12] B. Erol and S. Z. Gurbuz, "A kinect-based human micro-doppler simulator," IEEE Aerospace and Electronic Systems Magazine, vol. 30, no. 5, pp. 6-17, 2015.

[13] K. Chetty, G. E. Smith, and K. Woodbridge, "Through-the-wall sensing of personnel using passive bistatic wifi radar at standoff distances," IEEE Transactions on Geoscience and Remote Sensing, vol. 50, no. 4, pp. 1218-1226, 2011.

[14] Ni usrp 2921. [Online]. Available: http://sine.ni.com/nips/cds/view/ $\mathrm{p} /$ lang/en/nid/212995

[15] K. Simonyan and A. Zisserman, "Very deep convolutional networks for large-scale image recognition," arXiv preprint arXiv:1409.1556, 2014. 